Physical Sciences | Denis Fedyanin and Alexander Chkhartishvili

\section{Opinion dynamics and consensus in social networks}

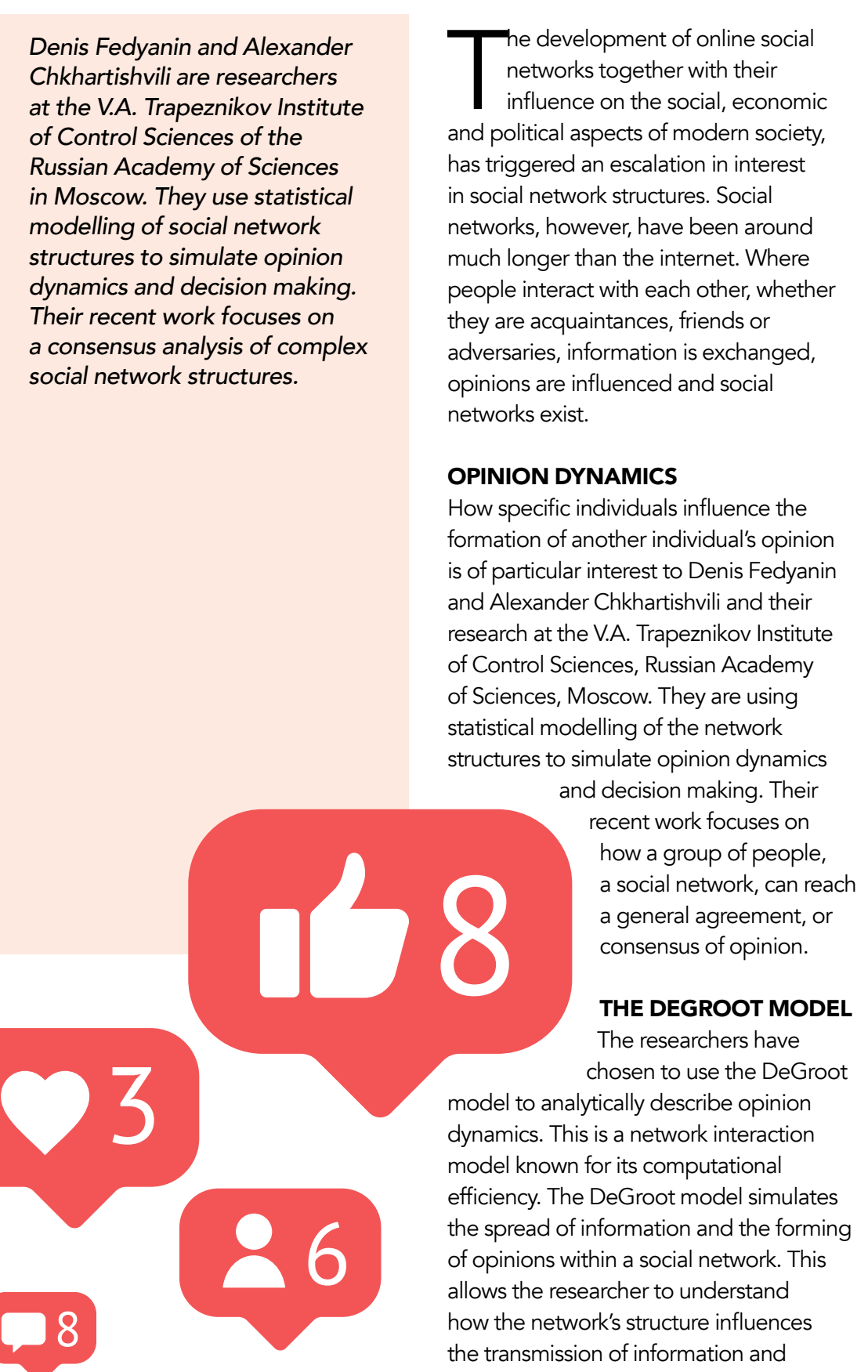

the formation of opinion. It also provides a description of how individual member

The DeGroot model is based on a Markov chain, a random process where the robability of any future event depends only on its current state, not on its past of the past. In this model, an agent is characterised by his or her opinion. Each opinion is allocated by an arbitrary real. number; not necessarily a probability s it would be with a Markov chain. The model pools the opinions of each agent into a network model, which

gives a prediction on the final opinions of all persons. These opinions coincid network consensus takes place. The model can also be used to determine how central an individual agent is with
network's decision making.

\section{MODELLING OPINION DYNAMICS}

\section{AND CONSENSUS}

incted as a node a network. Initially each agent has a specific opinion about an issue which is given a numerical value between 0 and 1 that can be a probability or have other interpretations. Opinions are updated over time. All opinions evolve in accordance with an iterative and linear process whereby each agent's opinion is updating using a weighted average of the other agents' opinions. These weights are based on influence and confidence. The level of confidence that agent $A$ has in agent $B$ is the same as the level of influence that agent $B$ has over agent $A$. The tota Agents comvof any agent is 1. Agents communicate by exchanging each agent's opinion is the weighted sum of the opinions of the agents that e or she trusts, where the weights are arises if opinions converge to a single value that is the overall opinion of the network. This is always the case when each person has non-zero confidence and there are no autonomous clusters in the network.

The contribution of each agent to the collective overall opinion can be determined using linear algebra

\section{REAL LIFE OPINIONS} information on their initial opinions construct model where and we new opinion is a combination of their last opinion and the opinions of their friends. Given this model we can measure the influence of each person in the group.

It is unlikely that each person just constructs their new opinion based on their previous opinion and the opinions of other people in the social group. It is more likely there are some hidden advisors that can also affect opinion.

\section{COMPOUND NODES}

edyanin and Chkhartishvili have modified the initial model to include the influence of these advisors in the formation of an individuals opinion. Where previously each person, or by a single agent, each person is now represented by a compound node
Let us consider a group of friends hhat form a social network. We have

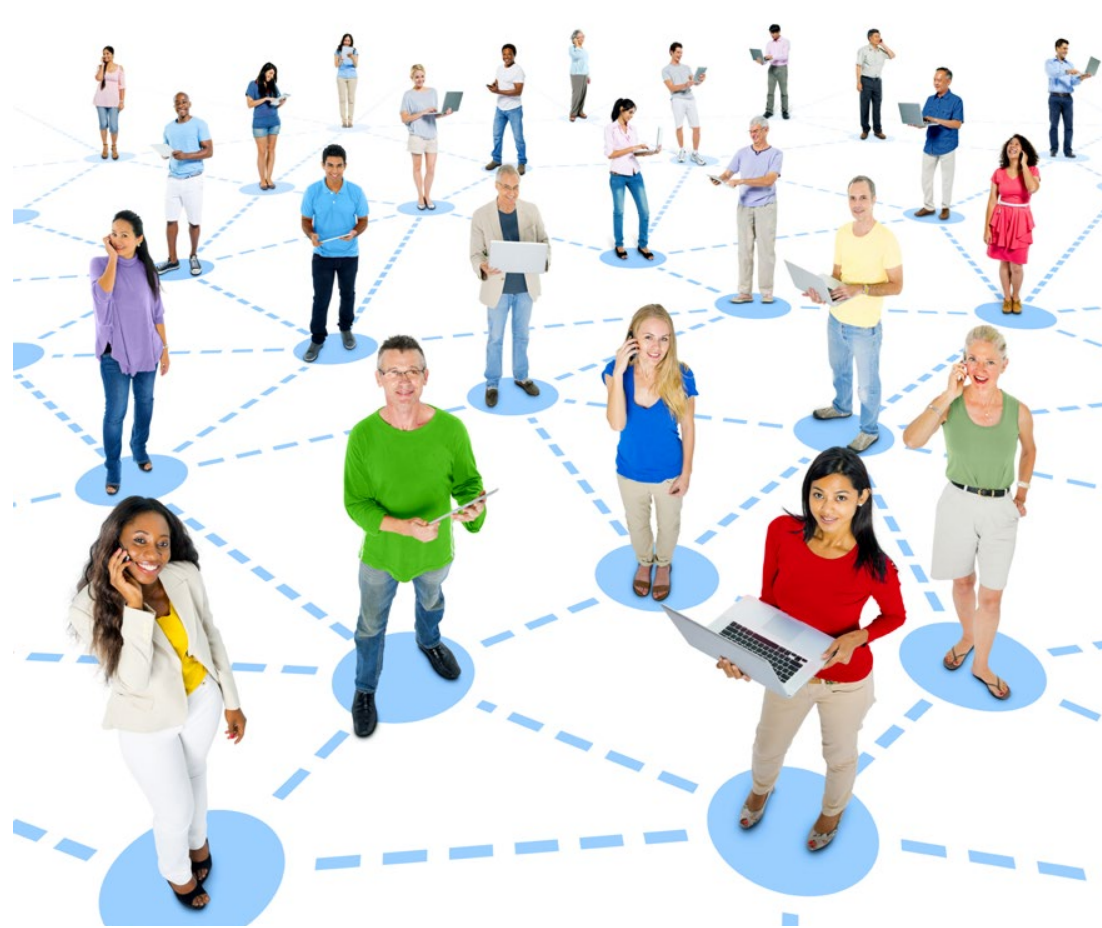

The development of online social networks has triggered an escalation in interest in social network structures.

comprising two agents, external and

CONSENSUS IN NETWORKS

OF COMPOUND NODES

Communication between a given node

and other network nodes is carried

out by the external agent. The interna

agent can only interact directly with

the extenal agent in the samenode, of a personal advisor.

The researchers have found that given

person in the initial network, they can calculate the new influences in a revised model by including a simple formula person respects his or her values and how their values could be changed by a person's behaviour.

If there is a consensus in the original model, then repetition of the iterative process over a sufficient period of time model and results in the formation of the collective overall opinion or consensus of all people in the group.

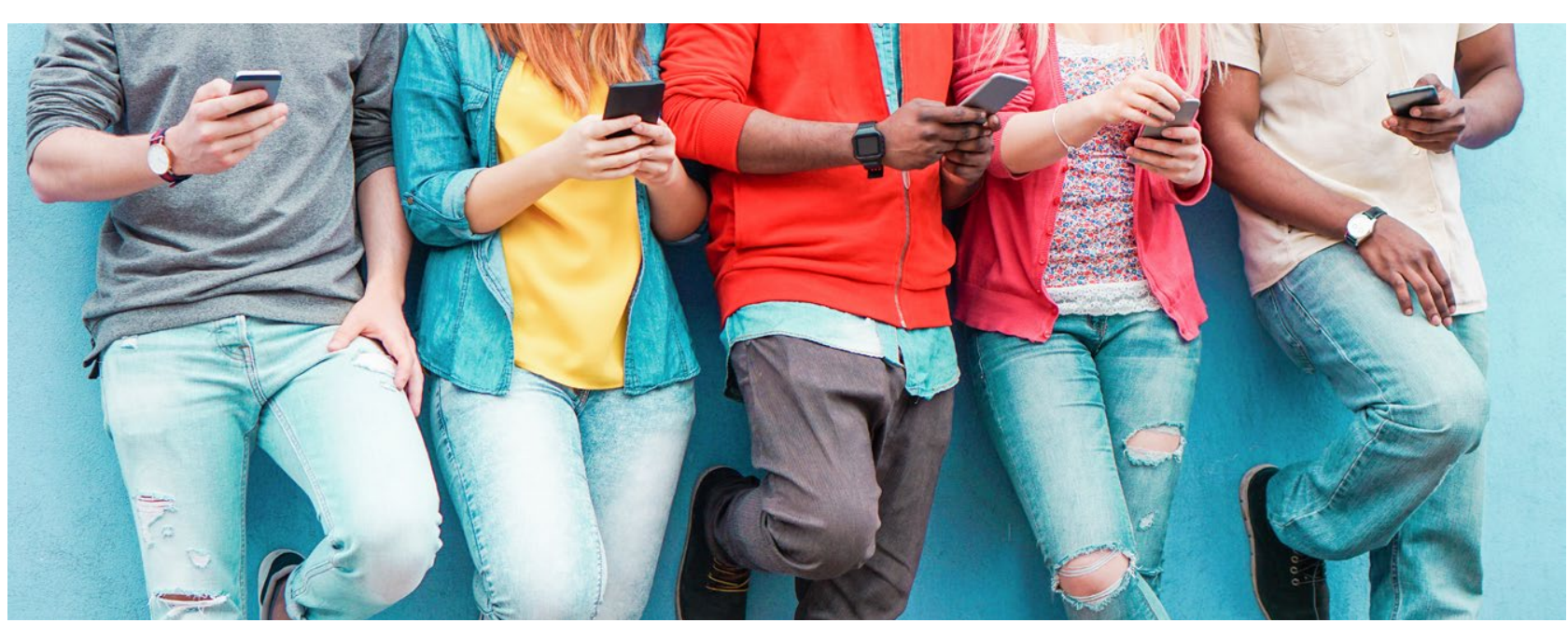




\section{MODELLING TWO}

\section{MMPOUND NODES}

The researchers have also studied

nodes and found that under certan

conditions a node's level of influence

can have a stronger dependence

on the internal parameter.

\section{APPLICATION}

The process can be applied to

the following situation. Ann and Bob

have to make a decision. Let us consider

two scenarios:

1: The decision is not very important,

so Ann and Bob do not involve

their advisors.

2: The decision is very important,

so Ann and Bob involve their advisors.

Someone's opinion is determined

by how much he or she wants to agree

These opinions are shown as colours.

in the diagram:

- Green represents 100\% agreement

with 'yes'.

- Red represents 0\% agreement with 'yes'

Arrows depict influences. The larger

the arrow from $A$ to $B$, the larger influence

of $A$ on $B$. Bob's influence on his advisors

is large but so is their influence on him.

Ann's influence on her advisors is small but

so is their influence on her.

Opinion dynamics
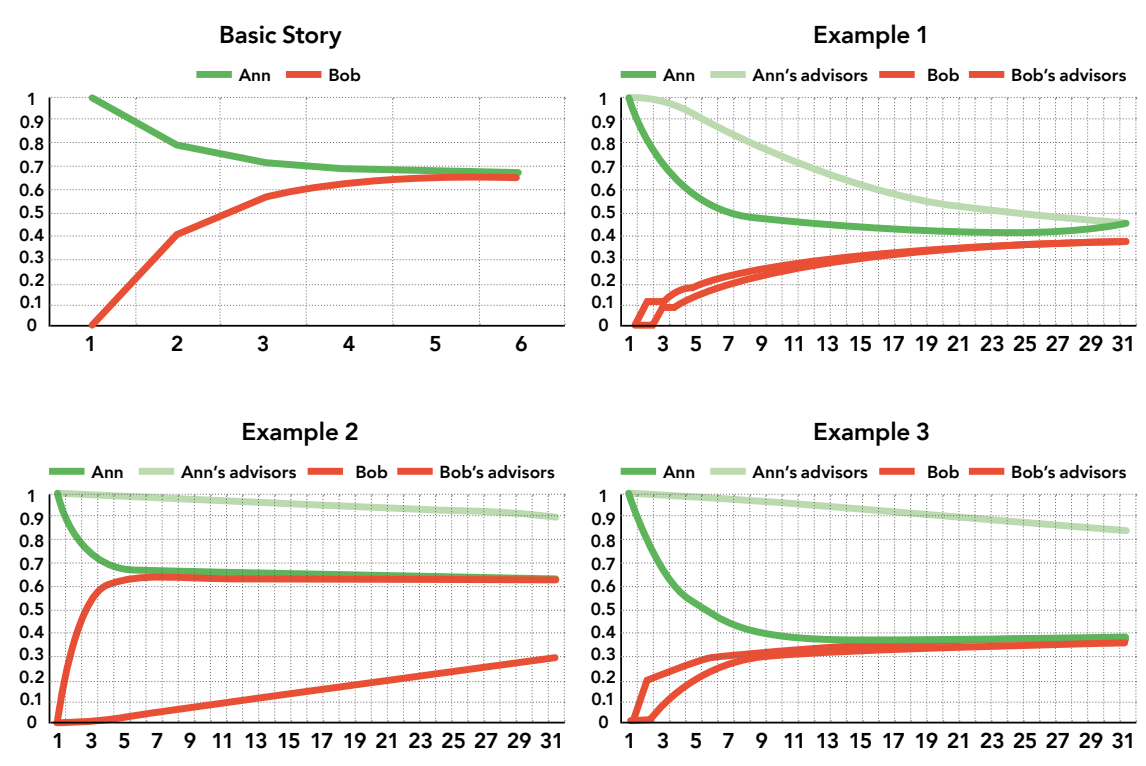

The horizontal axis represents time and the vertic
1 means $100 \%$ "Yes", and 0 means $100 \%$ "No".

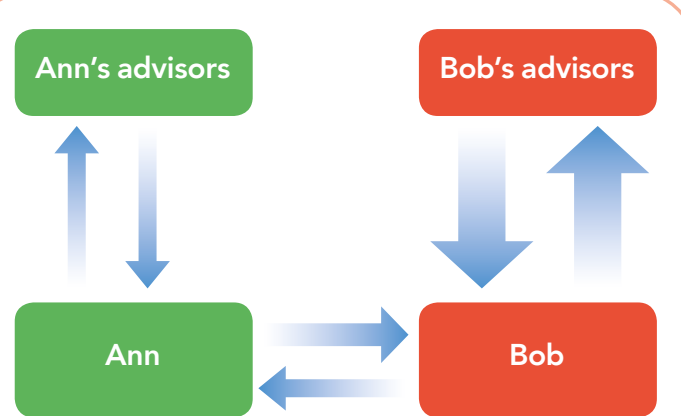

Social Network with Advisors

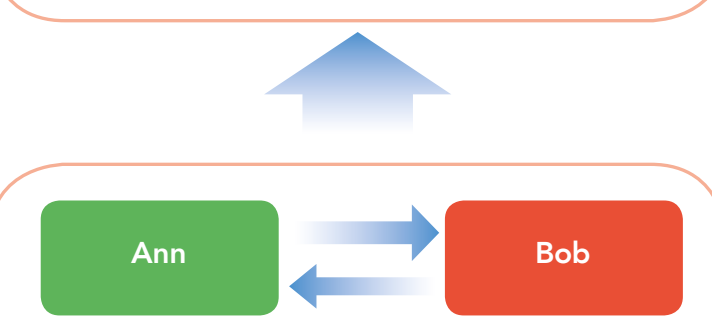

Social Network with NO Advisors

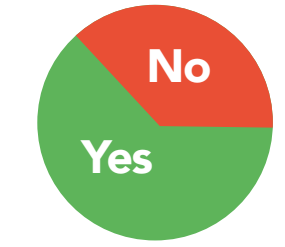

They have established a model that includes the overall influence of internal factors like confidence in personal advisors. Can we derive the consensus opinion in a setting if we know the opinions in The DeGroot model is a powerful tool in have found a solution and stated it using a simple formula

of information and beliefs It provides an explicit basis for measuring central iy within a social network and identifying e most influence over the opinions of a group.

Fedyanin and Chkhartishvili have extended the DeGroot model to enable consensus analysis of complex network sructures where each node comprises two mutually interacting agents. They overall influence of internal factors on their initial opinions, without any restrictions on the number of members in the social network or the number of interactions

The researchers have identified further research involving the examination of the communication processes of nodes with more complicated internal structure, i.e. nodes where more than two agents interact, as well as fur her complex mode

\section{Behind the Research \\ Denis Fedyanin \\ Alexander Chkhartishvili}

E: dfedyanin@inbox.ru T: $\pm 7(926) 1771746 \quad T: \pm 7(465) 3348910$

Research Objectives

Denis Fedyanin and Alexander Chkhartishvili from the Russian Academy of Sciences model opinion dynamics parameters affect their social power.

\section{Detail}

Denis Fedyanin / V. A. Trapeznikov

Institute of Control Sciences of Russian Academy

of Sciences,

65 Profsoyuznaya street,

Moscow 117997,

Russia

Bio

Denis Fedyanin received his Master's Degree in Applied Mathematics and Physics from the Moscow Institute of Physics and Technology. He is a researcher with both the Active Systems Laboratory at the V.A. Trapeznikov Institute of Control Sciences, Russian Academy of Sciences, Moscow and the International Laboratory for Logic, Linguistics and Formal Philosophy at the Higher School

Dr Alexander Chkhartishvili has graduated from the Faculty of Computational Mathematics and Cybernetics of Science in Mascow State University. He is a Doctor of the Complex Networks laboratory atV.A. Trapeznikov Institute of Control Sciences, Russian Academy of Sciences, Moscow. He previously worked at the Faculty of Public Administration at Lomonosov Moscow State University.

Funding

V.A. Trapeznikov Institute of Control Sciences Social power, Compound nodes, DeGroot model.

\section{Collaborators}

- Burkor V.N.

- Novikor D.A.

- Gubanov D.A.

- Gubanov D.A.

\section{References}

Fedyanin D.N., Chkhartishvili A.G. (2018). 'Consensus in Social Networks of Compound Nodes'. Automation and Remote Control, Vol 79 (6), pp.1117-1124.

Fedyanin D.N., Chkhartishvili A.G. (2013). 'A model of informational control in active network structures in case .

Fedyanin D.N., Chkhartishvili A.G. (2011). 'On a model of informational control in social networks'. Automation and Remote Control, Vol 72 (10), pp.2181-2187.

Fedyanin D.N., Zuev A.S. (2012). 'Models of opinion control for agents in social networks.' Automation and Remote Control, Vol 73 (10), pp.1753-1764.

DeGroot, M.H. (1974). 'Reaching a Consensus'. Journal of the American Statistical Association, Vol 69 (345). pp.18-121.

\section{Personal Response}

\section{What motivated your interest in researching opinion}

II Opinions play an important role in our life. People decisions and make important changes in the world - such as decisions on global warming, acceptable lines between freedom and security, standards etc. These decisions could have an economic dimension too since they influence the production and sales of goods and, this knowledge could reasoning means that prediction of opinions deserves

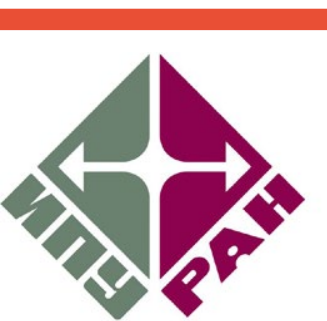

И Н С Т И Т У

ПРО БЛЕ М УПРАВЛЕНИЯ

им. B.A. TPA 